\title{
River surface flow estimation using a camera: a case study on the Tiber River
}

\author{
F. TAURO' ${ }^{1}$, M. PORFIRI ${ }^{1}$, A. PETROSELLI ${ }^{2}$, G. OLIVIERI $^{2}$, R. RAPITI ${ }^{2}$, \\ G. CIPOLLARI ${ }^{2}$ \& S. GRIMALDI ${ }^{1,3}$ \\ 1 Department of Mechanical and Aerospace Engineering, Polytechnic School of Engineering, New York University, \\ 6 MetroTech Center, Brooklyn, NY 11201, USA \\ 2 Department of Agriculture, Forests, Nature and Energy (DAFNE), University of Tuscia, Via San Camillo De Lellis \\ snc, 01100 Viterbo, Italy \\ 3 Department for Innovation in Biological, Agro-food and Forest systems (DIBAF), University of Tuscia, Via San \\ Camillo De Lellis snc, 01100 Viterbo, Italy \\ salvatore.grimaldi@,unitus.it
}

Monitoring surface water velocity during flood events is a challenging task. Techniques based on deploying instruments in the flow are often not feasible due to high velocity and abundant sediment transport. As a consequence, flow measurement campaigns are infrequent and stream velocity observations during major floods are scarce or absent.

In the last few years, large-scale particle image velocimetry (LSPIV) was proposed to overcome some of these drawbacks (Fujita et al. 1997). The novelty of LSPIV should be sought in its capacity of extracting desired kinematic information from a video of the surface streamflow. The general implementation of LSPIV can be summarized in three main sequential steps: video recording, image pre-processing, and image analysis. The video recording is the simplest phase, as it can be executed with a low-cost sport camera at a standard sampling frequency (30-60 frames per second $-\mathrm{fps})$ and at full-HD image resolution $(1920 \times 1080$ pixels $)$. An image pre-processing step is necessary for treating video frames before the image analysis. Typical pre-processing includes pixel calibration, frame correction and frame matching, to compensate for distortions and undesired mechanical vibrations. The treated frame sequence is ultimately processed through standard PIV algorithms that return the sought velocity maps. PIV (Adrian 1991) is based on the cross-correlation of pairs of consecutive frames, in which each frame is subdivided into interrogation windows that are translated on a pixel grid.

In this context, we have recently proposed a novel apparatus (Tauro et al. 2015), that includes a low-cost camera (GoPro Hero 3) installed on a telescopic hollow aluminium bar to maintain its axis orthogonal to the water surface. Its main novelty with respect to current LSPIV implementations is the use of two lasers that are installed at the ends of a 1-m pole connected to the bar. Such lasers create reference points in the field of view that are crucial for pixel calibration, which would otherwise require surveying through GPS or total stations.

In this work, the experimental apparatus is utilized to study 10-year return period flood events in the Tiber River in Rome, Italy. Three tests were executed in different illumination and weather conditions, from a bridge in the centre of Rome, where the historical hydrological Ripetta crosssection is located (Fig. 1(c)). Videos were recorded at full-HD resolution and $60 \mathrm{fps}$ for two minutes duration each, and they were subsequently reduced to VGA resolution and resampled to $30 \mathrm{fps}$, so that the resulting 3600 consecutive frames were spaced in time by $0.033 \mathrm{~s}$. The LSPIV algorithm was applied on a sequence of 500 frames, which was extracted to guarantee a sufficient transit of floating material. Figure 1(c) illustrates the velocity maps obtained from the three tests. Velocity values of each node represent the average of velocity values estimated on the available 499 pairs of consecutive frames. The most satisfactory results are those in the middle panel of Fig. 1(c), where homogenous velocity vectors are obtained and the kinematics around and before the bridge pier are well reproduced. This should be attributed to the favourable illumination and weather conditions of Test 2 and to the fact that the field of view was well centred within the river. The least satisfactory velocity map is the one corresponding to Test 1 and reported in the top panel of Fig. 1(c). Test1 was performed in the evening and during rainfall. Notably, the velocities 

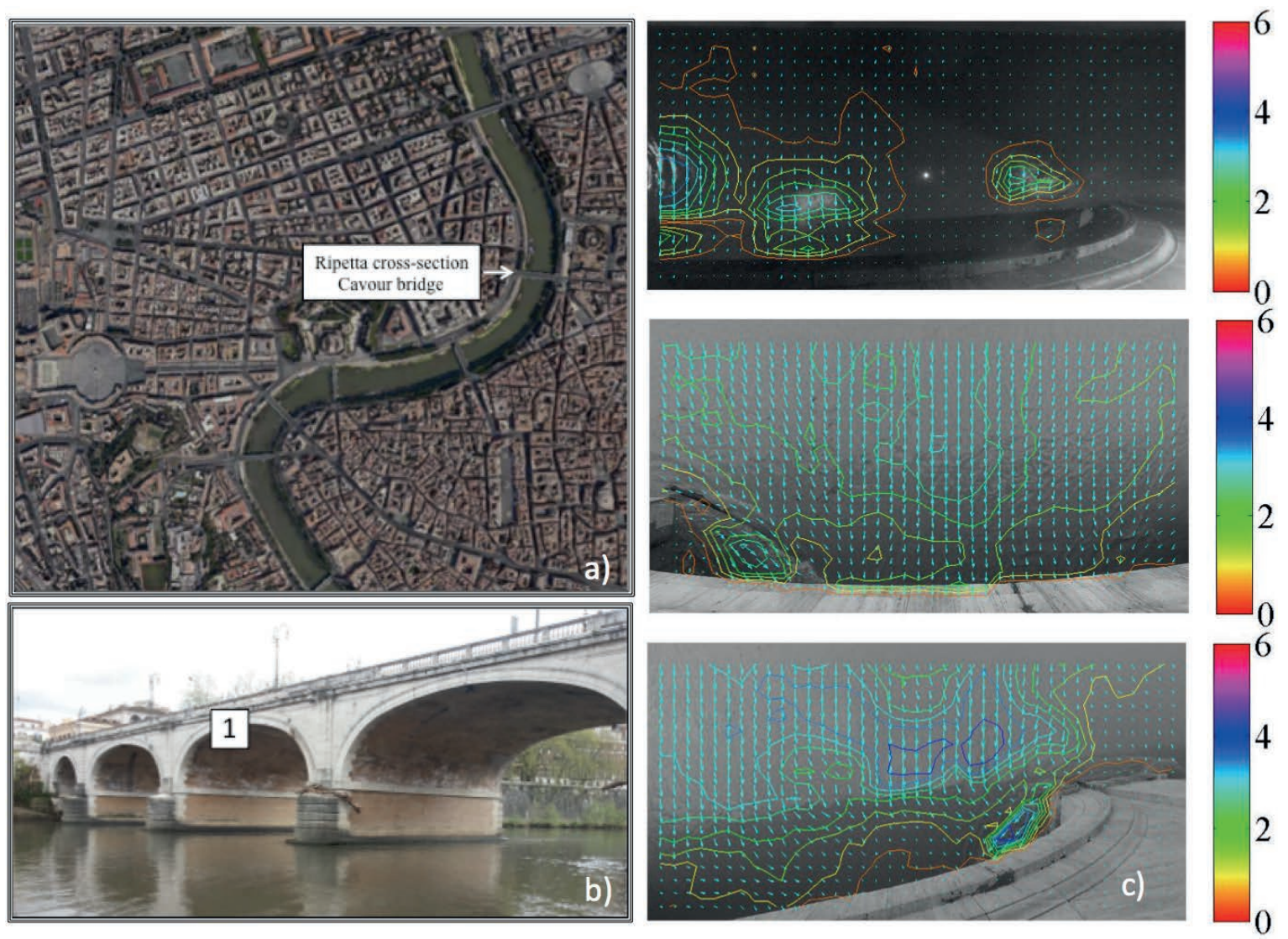

Fig. 1 (a) Map of the Tiber River in the Rome urban area, displaying the location of the hydrological cross-section. (b) View of Cavour Bridge; the experimental apparatus are located in "1". (c) Time averaged surface flow velocity maps for the three tests $(1,2$ and 3 from top to bottom). Values are in $\mathrm{m} / \mathrm{s}$.

estimated for Test1 pertain to a few artificially illuminated areas on the water surface in the proximity of the bridge.

Our findings indicate that future research should focus on devising a calibration procedure for identifying representative velocity values through salient indices of temporal and spatial variations. Furthermore, extensive analyses are necessary to establish criteria for selecting the duration of image sequences. In general, the proposed analysis confirms that LSPIV is a promising technique to aid river flow monitoring in challenging conditions.

Acknowledgements This work was supported by the Ministero degli Affari Esteri, Direzione Generale per la Promozione del Sistema Paese under the grant PGR00175; by the National Science Foundation under grant number BCS-1124795, by the Honors Center of Italian Universities, and by the American Geophysical Union Horton Research Grant for PhD students. The authors thank Francesco Mele, Domenico Spina, and Luigi D'Aquino from Ufficio Idrografico e Mareografico (UIM) at Regione Lazio, Italy, and Mirco Bartolini and Guido Bernardi from CAE spa for technical support.

\section{REFERENCES}

Adrian, R. J. (1991) Particle-imaging techniques for experimental fluid-mechanics. Annual Review of Fluid Mechanics 23, $261-304$. Fujita, I., Muste, M. and Kruger, A. (1997) Large-scale particle image velocimetry for flow analysis in hydraulic engineering applications. Journal of Hydraulic Research 36(3), 397-414.

Tauro, F., M. Porfiri, and S. Grimaldi (2014), Orienting the camera and firing lasers to enhance large scale particle image velocimetry for streamflow monitoring, Water Resour. Res., 50, doi:10.1002/2014WR015952. 